\title{
ARTERIES OF THE CERVICAL SPINAL CORD IN THE EUROPEAN HARE
}

\author{
Maženský, D., Flešárová S. \\ Department of Anatomy, Histology and Physiology \\ University of Veterinary Medicine and Pharmacy, Komenského 73, 04181 Košice \\ The Slovak Republic \\ david.mazensky@uvlf.sk
}

\section{ABSTRACT}

The aim of this study was to describe the blood supply to the cervical spinal cord in the European hare using the dissection technique. This study was carried out on 10 adult European hares. The arterial system of the cervical spinal cord was injected using Batson's corrosion casting kit No. 17. The presence of the ventral radicular branches of the rami spinales entering the ventral spinal artery in the cervical region was observed in $\mathbf{6 2 . 1} \%$ as left-sided and in $37.9 \%$ of the cases as right-sided. There were two dorsal spinal arteries located on the dorsal surface of the cervical spinal cord in $70 \%$ of the cases, and no dorsal spinal artery in $30 \%$ of the cases. The presence of the ventral radicular branches of the rami spinales that reached the spinal cord, was observed in $66.2 \%$ of the cases on the left side and in $33.8 \%$ of the cases on the right side. Based on our results, we can conclude that there is high variability of the blood supply to the cervical spinal cord in the European hare.

Key words: European hare; cervical spinal cord; dorsal spinal artery; ventral spinal artery

\section{INTRODUCTION}

The European hare is one of the most frequently occurring wild animals in Slovakia. Despite its relatively abundant occurrence, there is a significantly gap of information about its anatomy in the literature.

The unique anatomy of each region of the cervical spinal cord demands a study of this nature. The domesticated rabbit has been widely used as a laboratory animal to test the effects of neuroprotective drugs and to examine the pathophysiology of spinal cord injury and, for this reason, we studied the arterial arrangements of the cervical spinal cord in the rabbit [6]. The arterial supply to the cervical spinal cord has been described in several investigations $[1,3,10]$. Research on the arrangements and variability of the feeding arteries of the spinal cord in several species of laboratory animals $[4,9,11,12]$ and in man [7] are more common. Until now, the arterial arrangements of the cervical spinal cord in the European hare has not yet been described.

The aim of this study was to describe the blood supply of the European hare spinal cord with a focus on the cervi- 
cal section. We describe some variations in the arterial pattern of the segmental branches of the cervical spinal cord in the European hare.

\section{MATERIALS AND METHODS}

This study was carried out on 10 adult European hares (Lepus europaeus, L. 1758), aged 140 days. We used hares (obtained from ISFA APRC, Nitra, Slovak Republic) of both sexes (female $n=5$; male $n=5$ ) with a weight range between $1.5-1.8 \mathrm{~kg}$ in an accredited experimental laboratory of the University of Veterinary Medicine and Pharmacy in Kosice. The animals were kept in cages under standard conditions (temperature $15-20^{\circ} \mathrm{C}$, relative humidity $45 \%$, 12-hour light period), and fed with a granular feed mixture (O-10 NORM TYP, Spišské kŕmne zmesi, Spišské Vlachy, Slovak Republic). The drinking water was available to all animals ad libitum. The animals were injected intravenously with heparin (50000 IU. $\mathrm{kg}^{-1}$ ) $30 \mathrm{~min}$ before they were sacrificed by intravenous injection of embutramide (T-61, $\left.0.3 \mathrm{ml} . \mathrm{kg}^{-1}\right)$. Immediately after euthanasia, the vascular network was perfused with a physiological solution. During manual injection through the ascending aorta, the right atrium of the heart was opened in order to lower the pressure in the vessels to ensure an optimal injection distribution. Batson's corrosion casting kit No. 17, using a volume of $50 \mathrm{ml}$ (Dione, České Budějovice, Czech Republic) was used as the casting medium. After polymerization of the medium, $4 \%$ formaldehyde was injected into the vertebral canal between the occipital bone and the first cervical vertebra, and between the sixth and seventh cervical vertebra to fix the spinal cord. After 1-week of fixation, the vertebral canal was opened by removing the vertebral arches in the cervical spinal region. Also, the occipital bone was partly removed. The prepared spinal cords were fixed in $10 \%$ formaldehyde. This study was carried under authority decision No. 2647/07-221/5.

\section{RESULTS}

The ventral spinal artery was present as a single trunk located on the ventral surface of the spinal cord. On the dorsal surface, there were either two dorsal spinal arteries or no dorsal spinal artery at all. The ventral spinal artery had a rostral connection with the vertebral artery. The dorsal and ventral arteries received, along their course, several dorsal and ventral radicular branches entering the intervertebral foramina. Some radicular branches significantly contributed to the spinal cord blood supply.

The ventral spinal artery was located along the ventral median fissure of the spinal cord. Rami spinales arising from the bilateral vertebral arteries entered the vertebral canal through the intervertebral foramen. After entering the vertebral canal, they sent to the spinal cord, ventral and dorsal radicular branches. Some ventral radicular branches entered the ventral spinal artery. The frequency of occurrence of individual ventral radicular branches reaching the spinal cord is shown in Table 1 . The left-sided ventral radicular branches entering the ventral spinal artery were present in $62.1 \%$ of the cases, the right sided ventral radicular branches were present in $37.9 \%$ of the cases.

Two dorsal spinal arteries located on the dorsal surface of the cervical spinal cord were present in $70 \%$ of the cases (Fig. 1), and not any dorsal spinal artery in $30 \%$ of the cases (Fig. 2). Two longitudinal dorsal spinal arteries were formed by the fusion of the small cranial and caudal branches arising from the dorsal radicular branches of spinal arteries. We found no rostral origin of dorsal spinal arteries in the area of formation of the basilar artery. In the case of the absence of the dorsal spinal arteries, the cervical part of spinal cord received the blood by means of dorsal radicular branches of spinal arteries with very irregular arrangements (Fig. 2). The frequency of occurrence of individual dorsal radicular branches reaching the spinal cord is shown in Table 2. The left-sided dorsal radicular branches were present in $66.2 \%$ of the cases, the right-sided ventral radicular branches were present in $33.8 \%$ of the cases.

\section{DISCUSSION}

Based on our results, we can conclude that there is a high variability of the blood supply to the cervical part of the spinal cord in the European hare. Until now, the arterial blood supply of the cervical spinal cord in the European hare has not been described. In the study of cervical spinal cord arteries, dogs, rats, pigs and rabbits have often been used. In the dog, the blood supply of the cervical spinal cord have been studied in detail, with variations in the origin of the ventral spinal artery and the frequency of the occur- 
Table 1. Frequency of occurrence of ventral radicular arteries of the cervical spinal cord that contributed significantly to the spinal cord blood supply

Occurrence of arterial spinal branches [\%]

\begin{tabular}{ccc} 
Level & Right & Left \\
\hline C1 & 0 & 0 \\
C2 & 0 & 40 \\
C3 & 20 & 40 \\
C4 & 30 & 60 \\
C5 & 60 & 70 \\
C6 & 60 & 30 \\
C7 & 30 & 80 \\
C8 & 20 & 40 \\
\hline
\end{tabular}

C - Cervical segment of the spinal cord

Table 2. Frequency of occurrence of dorsal radicular arteries of the cervical spinal cord that contributed significantly to the spinal cord blood supply

Occurrence of arterial spinal branches [\%]

\begin{tabular}{ccc} 
Level & Right & Left \\
\hline C1 & 0 & 50 \\
C2 & 40 & 50 \\
C3 & 30 & 60 \\
C4 & 50 & 50 \\
C5 & 30 & 70 \\
C6 & 30 & 80 \\
C7 & 0 & 30 \\
C8 & 50 & 60 \\
\hline
\end{tabular}

C - Cervical segment of the spinal cord

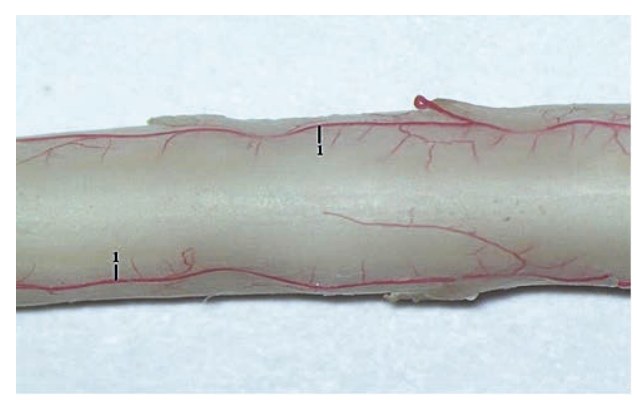

Fig. 1. Presence of two longitudinal dorsal spinal arteries (1) dorsal spinal artery. Dorsal view. Magn. $\times 12.5$

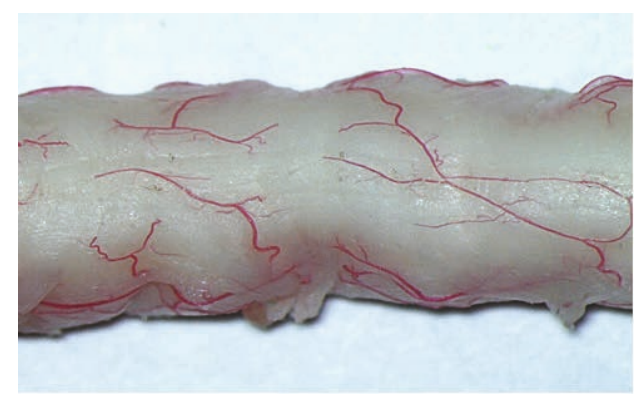

Fig. 2. Absence of dorsal spinal arteries Dorsal view. Magn. $\times 20$

rence of the spinal arteries described[8]. The blood supply of the rat spinal cord was probably the most profusely documented, but the results were often very different $[2,3$, $9,10,13]$. In the pig, only the variations and the presence of extrasegmental arteries in the spinal cord blood supply have been described [11]. The frequency of the occurrence of the segmental spinal arteries was higher on the left than on the right side; the opposite for the case in dogs [8]. The presence of ventral branches of spinal branches entering the ventral spinal artery in the cervical region was more frequently on the left than on the right side. A similar arrangement was found in the rabbit [6]. We did not find any connections of the dorsal spinal arteries in the area of formation of the basilar artery in our study. On the dorsal surface we found two longitudinal dorsal spinal arteries receiving dorsal branches of spinal arteries or they were absent. A similar situation was described in the rabbit [6]. The presence of dorsal branches of the spinal branches was more frequently on the left than on the right side, like in the rabbit [6]. 


\section{CONCLUSIONS}

Study of the arterial patterns of the spinal cord provides additional information concerning the manner of vascularization of the central nervous system in general $[5,13]$.

\section{REFERENCES}

1. Bilgen, M., Al-Hafez, B., 2006: Comparison of spinal vasculature in mouse and rat: investigations using MR angiography. Neuroanatomy, 5, 12-16.

2. Brightman, M. W., 1956: Comparative anatomy of spinal cord vasculature. Anat. Rec., 124, 264.

3. Koyanagi, I., Tator, CH., Lea, P. J., 1993: Three-dimensional analysis of the vascular system in the rat spinal cord with scanning electron microscopy of vascular corrosion casts. Part 1 : Normal spinal cord. Neurosurg., 33, 277-283.

4. Lang-Lazdunski, L., Matsushita, K., Hirt, L., Waeber, C., et al., 2000: Spinal cord ischemia. Development of a model in the mouse. Stroke, 31, 208-213.

5. Martirosyan, N. L., Feuerstein, J. S., Theodore, N., Cavalcanti, D. D., et al., 2011: Blood supply and vascular reactivity of the spinal cord under normal and pathological conditions. J. Neurosurg. Spine, 15, 238-251.

6. Mazensky, D., Danko, J., Petrovova, E., Luptakova, L., et al., 2012: Arterial arrangement of the cervical spinal cord in rabbit. Anat. Sci. Int., 87, 155-159.
7. Nijenhuis, R. J., Leiner, T., Cornips, E. M., 2004: Spinal cord feeding arteries at MR angiography for thoracoscopic spinal surgery: feasibility study and implications for surgical approach. Radiology, 233, 541-547.

8. Pais, D., Casal, D., Arantes, M., Casimiro, M., O’Neill, J. G., 2007: Spinal cord arteries in Canis familiaris and their variations: implications in experimental procedures. Braz. J. Morphol. Sci., 24, 224-228.

9. Schievink, W. I., Luyendijk, W., Los, J. A., 1988: Does the artery of Adamkiewicz exist in the albino rat? J. Anat., 161, 95-101.

10. Soutoul, J. H., Gouaz'e, A., Castaing, J., 1964: Les arte'res de la moelle epiniere des animaux d' experimentation. III.- etude comparative durat, cobaye, lapin, chat, chien, orang-outang, chimpanze, avec l'homme et le foetus. Pathol. Biol., 12, 950-962.

11. Strauch, J. T., Spielvogel, D., Lauten, A., Zhang, N., et al., 2003: Importance of extrasegmental vessels for spinal cord blood supply in a chronic porcine model. Eur. J. Cardiothorac. Surg., 24, 817-824.

12. Tveten, L., 1976: Spinal cord vascularity IV. The spinal cord arteries in the rat. Acta Radiol., 17, 385-398.

13. Woollam, D. H. M., Millen, J. W., 1955: The arterial supply of the spinal cord and its significance. J. Neurol. Neurosurg. Psychiatry, 18, 97-102.

Received January 22, 2016 